\title{
ON THE APPROXIMATE CONTROLLABILITY OF STACKELBERG-NASH STRATEGIES FOR STOKES EQUATIONS
}

\author{
F. GUILLÉN-GONZÁLEZ, F. MARQUES-LOPES, AND M. ROJAS-MEDAR \\ (Communicated by Walter Craig)
}

\begin{abstract}
We study a Stackelberg strategy subject to the evolutionary Stokes equations, considering a Nash multi-objective equilibrium (not necessarily cooperative) for the "follower players" (as they are called in the economy field) and an optimal problem for the leader player with approximate controllability objective.

We will obtain the following three main results: the existence and uniqueness of the Nash equilibrium and its characterization, the approximate controllability of the Stokes system with respect to the leader control and the associate Nash equilibrium, and the existence and uniqueness of the Stackelberg-Nash problem and its characterization.
\end{abstract}

\section{INTRODUCTION}

The use of the optimization problems has characterized an increase in the development of engineering or economy areas in the last few decades.

Initially, problems involving a unique objective were considered, but afterwards, more realistic situations with several (in general conflicting) objectives have been considered. Normally, in the classical mono-objective control problem, a functional adding the objectives of the problem is defined and a unique control is used. When it is not clear how to average the different objectives or when several controls are used, the introduction of the multi-objective problems is essential.

The different equilibrium notions for multi-objective problems were introduced in economics and games theory (see [14, [16, [13]).

In this work, we are interested in developing a Stackelberg-Nash strategy where the dynamics of the system is given by partial differential equations from fluid mechanics. We assume that we can act in the dynamics of a Stokes problem in velocity and pressure formulation by a hierarchy of controls. According to the formulation given by H. Von Stackelberg in 1934 [16], there are local controls that will be called followers and a global control called a leader. In fact, several followers are considered with their own objectives, and the leader has an approximate controllability objective admitting Nash equilibrium for the followers.

Received by the editors September 14, 2009 and, in revised form, September 15, 2011.

2010 Mathematics Subject Classification. Primary 76D55, 35Q30; Secondary 76D05, 93C20.

Key words and phrases. Stokes equations, approximate controllability, multi-objective optimization, Stackelberg-Nash strategies.

The first author was supported in part by the DGI-MEC Grant MTM2006-07932 (Spain), Junta de Andalucía project P06-FQM-02373 (Spain) and Fondecyt-Chile, Grant 1080628.

The third author was supported in part by the DGI-MEC Grant MTM2006-07932 (Spain) and Fondecyt-Chile, Grant 1080628. 
Some works related with this theme are the following. When linear parabolic differential equations are considered as constraints, Lions' works ([10, [11, [12]) present some results on Pareto and Stackelberg equilibrium. Díaz and Lions [2] studied the existence of Stackelberg-Nash equilibrium, and this study is extended by Díaz [3] given a characterization of the solution by means of Fenchel-Rockafellar duality theory. In 2 and [3], the followers and the leader have the same approximate controllability objective, the followers in a local manner and the leader globally.

On the other hand, Ramos, Glowinski and Periaux [8, 9] studied Nash equlibrium from the theoretical and numerical point of view, first for linear parabolic differential equations [8] and afterwards for the Burgers equation [9].

To our best knowledge, there are not any works on Stackelberg-Nash equilibrium associated to fluid mechanics. In this work, we will study the Stackelberg-Nash equilibrium with constraints given by the evolution Stokes equations; see [7] for a description of the problem.

For simplicity, we consider two (local) control functions $\left(\mathbf{v}^{(1)}, \mathbf{v}^{(2)}\right)$ acting in the system, distributed in two open regions $\omega_{1}$ and $\omega_{2}$ respectively, a (global) control $\mathbf{f}$ distributed on the region $\omega$ (containing in particular a $\omega_{1} \cup \omega_{2}$ ) and an associated state $\mathbf{u}$ considered as the solution of the Stokes system with previous distributed controls.

We consider that the objective of the leader is of controllability type. In fact, the leader want to drive the state velocity $\mathbf{u}$ at final time $T$ "very closed" (in the $\mathbf{L}^{2}(\Omega)$-sense) of a wished state $\mathbf{u}_{T}$, without a big cost for the control $\mathbf{f}$. On the other hand, the main objective of each follower acting on the control $\mathbf{v}^{(i)}(i=1,2)$ is to hold the state $\mathbf{u}$ near a desired state $\mathbf{u}_{i, d}$ for all time $t \in(0, T)$ in an observability region $\omega_{i, d}$, without a big cost for the control $\mathbf{v}^{(i)}$.

In order to combine these two different objectives, we introduce the following strategy of Stackelberg-Nash type. Given $\mathbf{f}$ acting in $\omega$, we consider a Nash equilibrium for the multi-objective control problem for the followers $\mathbf{v}^{(i)}, i=1,2$, acting in $\omega_{i}$. Afterwards, we will consider the (mono-objective) control problem with respect to $\mathbf{f}$ subject to the objective of approximate controllability type. Since each follower has its own objective, a global control for the leader (acting at the least in all control subdomains of the followers) will be necessary in order to achieve the approximate controllability objective. This is written as the following hypothesis:

$$
\omega_{1} \cup \omega_{2} \subset \omega
$$

In this paper, we will obtain the following three main results: the existence and uniqueness of the Nash equilibrium and its characterization, the approximate controllability of the Stokes system with respect to the leader control and the associated Nash equilibrium, and the existence and uniqueness of the StackelbergNash problem.

The rest of the paper is organized as follows. In Section 2 the formulation of the problem is established. The existence and uniqueness of the Nash equilibrium is deduced in Section 3 jointly with its characterization. In Section 4, we solve the approximate controllability problem with respect to the leader control. Finally, the existence and uniqueness of the Stackelberg-Nash problem is deduced via a duality argument in Section 5. 


\section{Problem Formulation}

Let $\Omega$ be a bounded domain of $\mathbb{R}^{d}, d=2$ or 3 , with boundary $\partial \Omega$ of class $C^{1,1}$ (because $H^{2}(\Omega)$ regularity for the Stokes problem will be applied), $\omega$ an open subset of $\Omega$ (the control domain), and $\omega_{1}, \omega_{2}$ open subsets of $\omega$ (i.e. $\omega_{1}, \omega_{2} \subset \omega$ ) the secondary control domains. In order to consider a (possibly noncooperative) Nash equilibrium, it is usual to consider $\omega_{1} \cap \omega_{2}=\emptyset$ (although it is not necessary for the mathematical problem). Finally, let $\omega_{1, d}, \omega_{2, d}$ be open subsets of $\Omega$, representing the observability domains for the followers, which are localized in an arbitrary manner in $\Omega$.

We denote by $\mathbf{H}$ and $\mathbf{V}$ the spaces of types $L^{2}$ and $H^{1}$ respectively, associated to the incompressibility and adherence velocity conditions:

$$
\begin{aligned}
& \mathbf{H}=\left\{\mathbf{u} \in \mathbf{L}^{2}(\Omega): \nabla \cdot \mathbf{u}=0, \mathbf{u} \cdot \mathbf{n}_{\mid \partial \Omega}=0\right\}, \\
& \mathbf{V}=\left\{\mathbf{u} \in \mathbf{H}^{1}(\Omega): \nabla \cdot \mathbf{u}=0, \mathbf{u}_{\mid \partial \Omega}=0\right\} .
\end{aligned}
$$

Observe that the boldface is used to denote the vectorial spaces in order to distinguish from the scalar ones. For instance, $\mathbf{L}^{2}(\Omega)=L^{2}(\Omega)^{d}$.

We will consider the two (secondary) objective functionals $(i=1,2)$

$$
J_{i}\left(\mathbf{f}, \mathbf{v}^{(1)}, \mathbf{v}^{(2)}\right)=\frac{\alpha_{i}}{2} \int_{0}^{T} \int_{\omega_{i, d}}\left|\mathbf{u}-\mathbf{u}_{i, d}\right|^{2} d x d t+\frac{\mu_{i}}{2} \int_{0}^{T} \int_{\omega_{i}}\left|\mathbf{v}^{(i)}\right|^{2} d x d t
$$

and the (main) objective functional

$$
J(\mathbf{f})=\frac{1}{2} \int_{0}^{T} \int_{\omega}|\mathbf{f}|^{2} d x d t
$$

where $\mu_{i}>0, \alpha_{i}>0$ are constants, $\mathbf{u}_{i, d} \in L^{2}\left(\omega_{i, d} \times(0, T)\right)$ are given functions, the function $\mathbf{f} \in L^{2}(\omega \times(0, T))$ is the (leader) control and $\mathbf{v}^{(i)} \in L^{2}\left(\omega_{i} \times(0, T)\right)$ are the (follower) controls. In (2.1) the state $\mathbf{u}$ is defined as the strong solution of the evolution Stokes problem (for instance, see [17])

$$
\left\{\begin{array}{l}
\partial_{t} \mathbf{u}-\nu \Delta \mathbf{u}+\nabla p=\mathbf{g}+\mathbf{f} \chi_{\omega}+\mathbf{v}^{(1)} \chi_{\omega_{1}}+\mathbf{v}^{(2)} \chi_{\omega_{2}}, \text { in } Q \\
\operatorname{div} \mathbf{u}=0, \text { in } Q ; \\
\mathbf{u}(0)=\mathbf{u}_{0}, \text { in } \Omega ;\left.\mathbf{u}\right|_{\Sigma}=0
\end{array}\right.
$$

(i.e. $\mathbf{u} \in L^{\infty}(0, T ; \mathbf{V}) \cap L^{2}\left(0, T ; \mathbf{H}^{2}\right), \partial_{t} \mathbf{u} \in L^{2}(0, T ; \mathbf{H})$ and $\left.p \in L^{2}\left(0, T ; H^{1}\right)\right)$, where we denote $Q=(0, T) \times \Omega$ and $\Sigma=(0, T) \times \partial \Omega$. Also observe that for instance $\mathbf{f} \chi_{\omega}$ denotes the extension of $\mathbf{f}$ by zero from $\omega$ to the whole domain $\Omega$.

Here, $\mathbf{u}$ is the velocity of the fluid, $p$ is the pressure, $\nu>0$ is the (constant) viscosity coefficient and $\mathbf{u}_{0} \in \mathbf{V}$ and $\mathbf{g} \in L^{2}\left(0, T ; \mathbf{L}^{2}(\Omega)\right)$ are given functions (the initial velocity and the external force respectively). Since there exists a unique strong solution of (2.3) , we can denote in the state $\mathbf{u}$ the dependence on the controls implicitly as follows:

$$
\mathbf{u}=\mathbf{u}\left(\mathbf{x}, t ; \mathbf{f}, \mathbf{v}^{(1)}, \mathbf{v}^{(2)}\right) .
$$

Functionals $J_{i}(i=1,2)$ measure the difference between the state velocity $\mathbf{u}$ and a prescribed velocity $\mathbf{u}_{i, d}$ in the observability domain of interest $\omega_{i, d}(i=1,2)$.

2.1. The control problem: Find control $\left(\mathbf{f}, \mathbf{v}^{(1)}, \mathbf{v}^{(2)}\right)$ and the corresponding state $\mathbf{u}$ verifying (2.3) and the Nash equilibrium related to the functionals $J_{i}$ defined in (2.1), minimizing the functional $J$ defined in (2.2) subject to the following 
(approximate) controllability constraint:

$$
\mathbf{u}\left(\cdot, T ; \mathbf{f}, \mathbf{v}^{(1)}, \mathbf{v}^{(2)}\right) \in B_{L^{2}}\left(\mathbf{u}_{T} ; \alpha\right),
$$

where $\mathbf{u}_{T}$ is a given function in $\mathbf{L}^{2}(\Omega)$ and $B_{L^{2}}\left(\mathbf{u}_{T} ; \alpha\right)$ denotes the ball of $\mathbf{L}^{2}(\Omega)$ with center in $\mathbf{u}_{T} \in \mathbf{L}^{2}(\Omega)$ and ratio $\alpha>0$ (a given number).

To explain this optimal problem, we are going to consider the following two subproblems:

- Problem 1. For any fixed leader control f find follower controls $\left(\overline{\mathbf{v}}^{(1)}, \overline{\mathbf{v}}^{(2)}\right)$ (depending on $\mathbf{f}$ ) and the associated state $\mathbf{u}=\mathbf{u}\left(\mathbf{f}, \overline{\mathbf{v}}^{(1)}(\mathbf{f}), \overline{\mathbf{v}}^{(2)}(\mathbf{f})\right)$ solution of (2.3) satisfying the Nash equilibrium related to $\left(J_{1}, J_{2}\right)$ defined in (2.1). That is, given $\mathbf{f}$, find $\left(\overline{\mathbf{v}}^{(1)}, \overline{\mathbf{v}}^{(2)}\right)$ such that

$$
\begin{aligned}
& J_{1}\left(\mathbf{f}, \overline{\mathbf{v}}^{(1)}, \overline{\mathbf{v}}^{(2)}\right)=\min _{\mathbf{v}^{(1)}} J_{1}\left(\mathbf{f}, \mathbf{v}^{(1)}, \overline{\mathbf{v}}^{(2)}\right), \\
& J_{2}\left(\mathbf{f}, \overline{\mathbf{v}}^{(1)}, \overline{\mathbf{v}}^{(2)}\right)=\min _{\mathbf{v}^{(2)}} J_{2}\left(\mathbf{f}, \overline{\mathbf{v}}^{(1)}, \mathbf{v}^{(2)}\right) .
\end{aligned}
$$

From the convexity of $J_{1}$ and $J_{2}$, one has that $\left(\overline{\mathbf{v}}^{(1)}, \overline{\mathbf{v}}^{(2)}\right)$ is a Nash equilibrium with respect to $\left(J_{1}, J_{2}\right)$ if and only if

$$
\begin{gathered}
\left\langle\frac{\partial J_{1}}{\partial \mathbf{v}^{(1)}}\left(\mathbf{f}, \overline{\mathbf{v}}^{(1)}, \overline{\mathbf{v}}^{(2)}\right), \mathbf{v}^{(1)}\right\rangle=0, \quad \forall \mathbf{v}^{(1)} \in L^{2}\left(\omega_{1} \times(0, T)\right), \\
\left\langle\frac{\partial J_{2}}{\partial \mathbf{v}^{(2)}}\left(\mathbf{f}, \overline{\mathbf{v}}^{(1)}, \overline{\mathbf{v}}^{(2)}\right), \mathbf{v}^{(2)}\right\rangle=0, \quad \forall \mathbf{v}^{(2)} \in L^{2}\left(\omega_{2} \times(0, T)\right) .
\end{gathered}
$$

The minimization problems (2.4) and (2.5), considered in an independent manner, are well-posed owing to the term $\frac{\mu_{i}}{2} \int_{0}^{T} \int_{\omega_{i}}\left|\mathbf{v}^{(i)}\right|^{2} d x d t$ in the cost functional $J_{i}$ which introduces any a priori constraints on the size of the control $\mathbf{v}^{(i)}$.

- Problem 2. After finding the controls $\overline{\mathbf{v}}^{(1)}=\overline{\mathbf{v}}^{(1)}(\mathbf{f}), \overline{\mathbf{v}}^{(2)}=\overline{\mathbf{v}}^{(2)}(\mathbf{f})$ and the state $\mathbf{u}=\mathbf{u}\left(\mathbf{f}, \overline{\mathbf{v}}^{(1)}(\mathbf{f}), \overline{\mathbf{v}}^{(2)}(\mathbf{f})\right)$ solution of (2.6)-(2.7) for each $\mathbf{f}$, we will look for an optimal control $\overline{\mathbf{f}}$ such that

$$
J(\overline{\mathbf{f}})=\min _{\mathbf{f}} J\left(\mathbf{f}, \overline{\mathbf{v}}^{(1)}(\mathbf{f}), \overline{\mathbf{v}}^{(2)}(\mathbf{f})\right)
$$

subject to the restriction of the approximate controllability type

$$
\mathbf{u}\left(\cdot, T ; \mathbf{f}, \overline{\mathbf{v}}^{(1)}(\mathbf{f}), \overline{\mathbf{v}}^{(2)}(\mathbf{f})\right) \in B_{L^{2}}\left(\mathbf{u}_{T}, \alpha\right) .
$$

\section{On the EXISTEnCE AND Uniqueness of the NASh EQUILIBRium}

Firstly, we are going to rewrite the system (2.6)-(2.7). For this, we consider the following functional spaces $\mathcal{H}_{i}=\mathbf{L}^{2}\left(\omega_{i} \times(0, T)\right), i=1,2, \mathcal{H}=\mathcal{H}_{1} \times \mathcal{H}_{2}$ and the (resolvent) operators

$$
L_{i} \in \mathcal{L}\left(\mathcal{H}_{i}, \mathbf{L}^{2}(Q)\right) \quad \text { defined as } \quad L_{i} \mathbf{v}^{(i)}=\mathbf{u}^{(i)},
$$

where $\mathbf{u}^{(i)} \in L^{2}\left(0, T ; \mathbf{H}^{2}\right) \cap L^{\infty}\left(0, T ; \mathbf{H}^{1}\right)$ is the (strong) solution of the Stokes problem:

$$
\left\{\begin{array}{l}
\partial_{t} \mathbf{u}^{(i)}-\nu \Delta \mathbf{u}^{(i)}+\nabla p^{(i)}=\mathbf{v}^{(i)} \chi_{\omega_{i}} \text { in } Q ; \\
\operatorname{div} \mathbf{u}^{(i)}=0, \text { in } Q ; \\
\mathbf{u}^{(i)}(0)=0, \text { in } \Omega,\left.\mathbf{u}^{(i)}\right|_{\Sigma}=0
\end{array}\right.
$$


Then for any $\mathbf{f} \in \mathbf{L}^{2}(\omega \times(0, T))$, we can write the solution of the state system (2.3) as

$$
\mathbf{u}=L_{1} \mathbf{v}^{(1)}+L_{2} \mathbf{v}^{(2)}+\mathbf{q}(\mathbf{f}),
$$

where $\mathbf{q}(\mathbf{f})$ is the solution of

$$
\left\{\begin{array}{l}
\partial_{t} \mathbf{q}-\nu \Delta \mathbf{q}+\nabla r=\mathbf{g}+\mathbf{f} \chi_{\omega}, \text { in } Q \\
\operatorname{div} \mathbf{q}=0, \text { in } Q ; \\
\mathbf{q}(0)=\mathbf{u}_{0}, \text { in } \Omega,\left.\mathbf{q}\right|_{\Sigma}=0
\end{array}\right.
$$

With this notation we can rewrite the functionals defined in (2.1) by

$$
\begin{aligned}
J_{i}\left(\mathbf{f}, \mathbf{v}^{(1)}, \mathbf{v}^{(2)}\right) & =\frac{\alpha_{i}}{2} \int_{0}^{T} \int_{\omega_{i, d}}\left|L_{1} \mathbf{v}^{(1)}+L_{2} \mathbf{v}^{(2)}-\mathbf{y}_{i, d}\right|^{2} d x d t \\
& +\frac{\mu_{i}}{2} \int_{0}^{T} \int_{\omega_{i}}\left|\mathbf{v}^{(i)}\right|^{2} d x d t \quad(i=1,2),
\end{aligned}
$$

where $\mathbf{y}_{i, d}=\mathbf{u}_{i, d}-\mathbf{q}(\mathbf{f})_{\mid \omega_{i, d}}$. Hence, $\left(\overline{\mathbf{v}}^{(1)}, \overline{\mathbf{v}}^{(2)}\right) \in \mathcal{H}$ is a Nash equilibrium if and only if

$$
\alpha_{i}\left(L_{1} \overline{\mathbf{v}}^{(1)}+L_{2} \overline{\mathbf{v}}^{(2)}-\mathbf{y}_{i, d}, L_{i} \mathbf{v}^{(i)}\right)_{\omega_{i, d} \times(0, T)}+\mu_{i}\left(\overline{\mathbf{v}}^{(i)}, \mathbf{v}^{(i)}\right)_{\omega_{i} \times(0, T)}=0,
$$

for each $i=1,2$ and for all pairs of controls $\left(\mathbf{v}^{(1)}, \mathbf{v}^{(2)}\right) \in \mathcal{H}$. It follows that

$$
\alpha_{i}\left(L_{i}^{*}\left[\left(L_{1} \overline{\mathbf{v}}^{(1)}+L_{2} \overline{\mathbf{v}}^{(2)}\right)_{\left.\right|_{\omega_{i, d}}}-\mathbf{y}_{i, d}\right], \mathbf{v}^{(i)}\right)_{\omega_{i} \times(0, T)}+\mu_{i}\left(\overline{\mathbf{v}}^{(i)}, \mathbf{v}^{(i)}\right)_{\omega_{i} \times(0, T)}=0,
$$

where $L_{i}^{*} \in \mathcal{L}\left(\mathbf{L}^{2}(Q), \mathcal{H}_{i}\right)$ is the adjoint operator of $L_{i}$. Consequently, we have

$$
\alpha_{i} L_{i}^{*}\left[\left(L_{1} \overline{\mathbf{v}}^{(1)}+L_{2} \overline{\mathbf{v}}^{(2)}\right) \chi_{\omega_{i, d}}\right]+\mu_{i} \overline{\mathbf{v}}^{(i)}=\alpha_{i} L_{i}^{*}\left(\mathbf{y}_{i, d}\right), \text { in } \mathcal{H}_{i} \quad(i=1,2) ;
$$

or equivalently, if we consider the operator $R=\left(R_{1}, R_{2}\right) \in \mathcal{L}(\mathcal{H}, \mathcal{H})$ defined as

$$
\forall \mathbf{v}=\left(\mathbf{v}^{(1)}, \mathbf{v}^{(2)}\right) \in \mathcal{H}, \quad R_{i} \mathbf{v}=\mu_{i} \mathbf{v}^{(i)}+\alpha_{i} L_{i}^{*}\left[\left(L_{1} \mathbf{v}^{(1)}+L_{2} \mathbf{v}^{(2)}\right) \chi_{\omega_{i, d}}\right], i=1,2,
$$

then $\overline{\mathbf{v}}=\left(\overline{\mathbf{v}}^{(1)}, \overline{\mathbf{v}}^{(2)}\right) \in \mathcal{H}$ is a Nash equilibrium iff $R \overline{\mathbf{v}}=\mathbf{z}:=\alpha_{i}\left(L_{i}^{*} \mathbf{y}_{i, d}(\mathbf{f})\right)_{i=1,2} \in$ $\mathcal{H}$. Moreover, for fixed $\left(\mathbf{u}_{1, d}, \mathbf{u}_{2, d}\right) \in \mathbf{L}^{2}\left(\omega_{1, d} \times(0, T)\right) \times \mathbf{L}^{2}\left(\omega_{2, d} \times(0, T)\right)$, the map

$$
\mathbf{f} \in \mathbf{L}^{2}(\omega \times(0, T)) \longrightarrow\left(L_{i}^{*} \mathbf{y}_{i, d}\right)_{i=1,2} \in \mathcal{H}
$$

is one-to-one, since this map is the composition of the (one-to-one) maps

$$
\mathbf{f} \longrightarrow \mathbf{q}(\mathbf{f}) \longrightarrow\left(\mathbf{y}_{i, d}\right)_{i=1,2}=\left(\mathbf{u}_{i, d}-\mathbf{q}(\mathbf{f}) \chi_{\omega_{i, d}}\right)_{i=1,2} \longrightarrow\left(L_{i}^{*} \mathbf{y}_{i, d}\right)_{i=1,2} .
$$

Proposition 3.1 (Existence and uniqueness of the Nash equilibrium). Assume that

$$
\alpha_{1}\left\|L_{2}\right\|^{2}<4 \mu_{2} \quad \text { and } \quad \alpha_{2}\left\|L_{1}\right\|^{2}<4 \mu_{1},
$$

where $\left\|L_{i}\right\|$ denotes the norm of the linear operator $L_{i}$ from $\boldsymbol{L}^{2}\left(\omega_{i} \times(0, T)\right)$ to $\boldsymbol{L}^{2}(Q)$. Then $R$ is an invertible operator. In particular, for each $\mathbf{f} \in \boldsymbol{L}^{2}(\omega \times(0, T))$, there exists a unique Nash equilibrium $\left(\overline{\mathbf{v}}^{(1)}(\mathbf{f}), \overline{\mathbf{v}}^{(2)}(\mathbf{f})\right)$ that is a solution of Problem 1.

Proof. We observe that

$$
(R \mathbf{v}, \mathbf{v})_{\mathcal{H}}=\sum_{i=1}^{2} \mu_{i}\left\|\mathbf{v}^{(i)}\right\|_{L^{2}\left(\omega_{i} \times(0, T)\right)}^{2}+\sum_{i=1}^{2} \alpha_{i}\left(\sum_{j=1}^{2} L_{j} \mathbf{v}^{(j)}, L_{i} \mathbf{v}^{(i)}\right)_{\omega_{i, d} \times(0, T)}
$$


where the last term of the right-hand side is computed as

$$
\begin{aligned}
\sum_{i=1}^{2} \alpha_{i} & \left(\sum_{j=1}^{2} L_{j} \mathbf{v}^{(j)}, L_{i} \mathbf{v}^{(i)}\right)_{L^{2}\left(\omega_{i, d} \times(0, T)\right)} \\
= & \alpha_{1}\left\|L_{1} \mathbf{v}^{(1)}\right\|_{L^{2}\left(\omega_{1, d} \times(0, T)\right)}^{2}+\alpha_{1}\left(L_{2} \mathbf{v}^{(2)}, L_{1} \mathbf{v}^{(1)}\right)_{\omega_{1, d} \times(0, T)} \\
& +\alpha_{2}\left(L_{1} \mathbf{v}^{(1)}, L_{2} \mathbf{v}^{(2)}\right)_{\omega_{2, d} \times(0, T)}+\alpha_{2}\left\|L_{2} \mathbf{v}^{(2)}\right\|_{L^{2}\left(\omega_{2, d} \times(0, T)\right)}^{2}
\end{aligned}
$$

Owing to the Young inequality, the cross terms can be bounded as

$$
\begin{aligned}
\alpha_{1}\left(L_{2} \mathbf{v}^{(2)}, L_{1} \mathbf{v}^{(1)}\right)_{\omega_{1, d} \times(0, T)} \geq & -\alpha_{1}\left\|L_{1} \mathbf{v}^{(1)}\right\|_{L^{2}\left(\omega_{1, d} \times(0, T)\right)}^{2} \\
& -\frac{\alpha_{1}}{4}\left\|L_{2} \mathbf{v}^{(2)}\right\|_{L^{2}\left(\omega_{1, d} \times(0, T)\right)}^{2}, \\
\alpha_{2}\left(L_{1} \mathbf{v}^{(1)}, L_{2} \mathbf{v}^{(2)}\right)_{\omega_{2, d} \times(0, T) \geq} & -\alpha_{2}\left\|L_{2} \mathbf{v}^{(2)}\right\|_{L^{2}\left(\omega_{2, d} \times(0, T)\right)}^{2} \\
& -\frac{\alpha_{2}}{4}\left\|L_{1} \mathbf{v}^{(1)}\right\|_{L^{2}\left(\omega_{2, d} \times(0, T)\right)}^{2} .
\end{aligned}
$$

Therefore, setting these bounds in (3.6), we obtain

$$
\begin{aligned}
(R \mathbf{v}, \mathbf{v})_{\mathcal{H}} \geq & \mu_{1}\left\|\mathbf{v}^{(1)}\right\|_{L^{2}\left(\omega_{1} \times(0, T)\right)}^{2}+\mu_{2}\left\|\mathbf{v}^{(2)}\right\|_{L^{2}\left(\omega_{2} \times(0, T)\right)}^{2} \\
& - \text { fraco }_{1} 4\left\|L_{2} \mathbf{v}^{(2)}\right\|_{L^{2}\left(\omega_{1, d} \times(0, T)\right)}^{2}-\frac{\alpha_{2}}{4}\left\|L_{1} \mathbf{v}^{(1)}\right\|_{L^{2}\left(\omega_{2, d} \times(0, T)\right)}^{2} \\
\geq & \mu_{1}\left\|\mathbf{v}^{(1)}\right\|_{L^{2}\left(\omega_{1} \times(0, T)\right)}^{2}+\mu_{2}\left\|\mathbf{v}^{(2)}\right\|_{L^{2}\left(\omega_{2} \times(0, T)\right)}^{2} \\
& -\frac{\alpha_{1}}{4}\left\|L_{2}\right\|^{2}\left\|\mathbf{v}^{(2)}\right\|_{L^{2}\left(\omega_{2} \times(0, T)\right)}^{2}-\frac{\alpha_{2}}{4}\left\|L_{1}\right\|^{2}\left\|\mathbf{v}^{(1)}\right\|_{L^{2}\left(\omega_{1} \times(0, T)\right)}^{2}
\end{aligned}
$$

By using hypotheses (3.5), we have

$$
(R \mathbf{v}, \mathbf{v})_{\mathcal{H}} \geq \gamma\|\mathbf{v}\|_{\mathcal{H}}^{2}, \text { for } \gamma=\min \left\{\mu_{1}-\frac{\alpha_{2}}{4}\left\|L_{1}\right\|^{2}, \mu_{2}-\frac{\alpha_{1}}{4}\left\|L_{2}\right\|^{2}\right\}>0 .
$$

Now, we define the functional $a: \mathcal{H} \times \mathcal{H} \rightarrow \mathbb{R}$ by

$$
a(\mathbf{v}, \mathbf{w})=(R \mathbf{v}, \mathbf{w})_{\mathcal{H}} .
$$

Obviously, from the definition of the operator $R$ and the inequality (3.8), $a(\cdot, \cdot)$ is a coercive, continuous bilinear form. Consequently, the conclusion follows from the Lax-Milgram Theorem.

Remark 3.2. The previous result can be generalized to an arbitrary number $N$ of local controls $\mathbf{v}^{(i)}$ and $N$ local objectives $J_{i}$, changing the hypothesis (3.5) by

$$
(N-1)\left(\sum_{j \neq i} \alpha_{j}\right)\left\|L_{i}\right\|^{2}<4 \mu_{i}, \quad \forall i=1, \ldots, N .
$$

For this, it is sufficient to develop the sum

$$
\sum_{i=1}^{N} \alpha_{i}\left(\sum_{j=1}^{N} L_{j} \mathbf{v}^{(j)}, L_{i} \mathbf{v}^{(i)}\right)_{\omega_{i, d} \times(0, T)}
$$


and to use the Young inequality in order to prove the coercivity of the operator $R$. Indeed, instead of (3.8), we now have

$$
\begin{aligned}
& \sum_{i=1}^{N} \mu_{i}\left\|\mathbf{v}^{(i)}\right\|^{2}-\frac{N-1}{4} \sum_{i=1}^{N} \alpha_{i}\left(\sum_{j \neq i}\left\|L_{j}\right\|^{2}\left\|\mathbf{v}^{(j)}\right\|^{2}\right) \\
= & \sum_{i=1}^{N} \mu_{i}\left\|\mathbf{v}^{(i)}\right\|^{2}-\frac{N-1}{4} \sum_{i=1}^{N}\left\|L_{i}\right\|^{2}\left\|\mathbf{v}^{(i)}\right\|^{2}\left(\sum_{j \neq i} \alpha_{j}\right) .
\end{aligned}
$$

Corollary 3.3 (Characterization of the Nash equilibrium). Given $\mathbf{f} \in \boldsymbol{L}^{2}(\boldsymbol{\omega} \times$ $(0, T))$, the pair $\left(\overline{\mathbf{v}}^{(1)}, \overline{\mathbf{v}}^{(2)}\right)$ is a Nash equilibrium of Problem 1 if and only if $\overline{\mathbf{v}}^{(i)}=$ $-\frac{1}{\mu_{i}} \mathbf{q}^{(i)} \chi_{\omega_{i}}, i=1,2$, where $\left(\mathbf{u}, \mathbf{q}^{(1)}, \mathbf{q}^{(2)}\right)$ is the solution of the coupled system

$$
\left\{\begin{array}{l}
\partial_{t} \mathbf{u}-\nu \Delta \mathbf{u}+\nabla p=\mathbf{g}+\mathbf{f} \chi_{\omega}-\frac{1}{\mu_{1}} \mathbf{q}^{(1)} \chi_{\omega_{1}}-\frac{1}{\mu_{2}} \mathbf{q}^{(2)} \chi_{\omega_{2}}, \text { in } Q \\
-\partial_{t} \mathbf{q}^{(i)}-\nu \Delta \mathbf{q}^{(i)}+\nabla r_{i}=\alpha_{i}\left(\mathbf{u}-\mathbf{u}_{i, d}\right) \chi_{\omega_{i, d}} \text { in } Q \quad(i=1,2) \\
\operatorname{div} \mathbf{u}=0, \text { in } Q ; \quad \operatorname{div} \mathbf{q}^{(i)}=0, \text { in } Q \quad(i=1,2) \\
\mathbf{u}(0)=\mathbf{u}_{0}, \text { in } \Omega,\left.\mathbf{u}\right|_{\Sigma}=0, \\
\mathbf{q}^{(i)}(T)=0, \text { in } \Omega,\left.\mathbf{q}^{(i)}\right|_{\Sigma}=0 \quad(i=1,2)
\end{array}\right.
$$

Notice that Corollary 3.3 implies in particular the existence and uniqueness of problem (3.10), which is a coupled problem of elliptic type with respect to the vectorial variable $(t, \mathbf{x})$.

Proof. We observe that characterization (2.6)-(2.7) of the Nash equilibrium of Problem 1 can be written as (3.2), i.e.: the pair $\left(\overline{\mathbf{v}}^{(1)}, \overline{\mathbf{v}}^{(2)}\right)$ is a Nash equilibrium if and only if

$$
\alpha_{i}\left(\mathbf{u}-\mathbf{u}_{i, d}, \mathbf{z}^{(i)}\right)_{\omega_{i, d} \times(0, T)}+\mu_{i}\left(\overline{\mathbf{v}}^{(i)}, \mathbf{v}^{(i)}\right)_{\omega_{i} \times(0, T)}=0 \quad(i=1,2),
$$

for any $\mathbf{v}^{(i)} \in \mathbf{L}^{2}\left(\omega_{i} \times(0, T)\right)$, where $\mathbf{z}^{(i)}$ is the strong solution of the Stokes problem

$$
\left\{\begin{array}{l}
\partial_{t} \mathbf{z}^{(i)}-\nu \Delta \mathbf{z}^{(i)}+\nabla p_{i}=\mathbf{v}^{(i)} \chi_{\omega_{i}} \text { in } Q ; \\
\operatorname{div} \mathbf{z}^{(i)}=0, \text { in } Q ; \\
\mathbf{z}^{(i)}(0)=0, \text { in } \Omega,\left.\quad \mathbf{z}^{(i)}\right|_{\Sigma}=0
\end{array}\right.
$$

Notice that (3.12) is the derivative of the state problem (2.3) with respect to $\mathbf{v}^{(i)}$.

To express (3.11) only in functions of $\mathbf{v}^{(i)}$, we introduce the adjoint states $\mathbf{q}^{(i)}(i=$ $1,2)$, as the strong solution of the problems

$$
\left\{\begin{array}{l}
-\partial_{t} \mathbf{q}^{(i)}-\nu \Delta \mathbf{q}^{(i)}+\nabla r_{i}=\alpha_{i}\left(\mathbf{u}-\mathbf{u}_{i, d}\right) \chi_{\omega_{i, d}} \text { in } Q ; \\
\operatorname{div} \mathbf{q}^{(i)}=0, \text { in } Q ; \\
\mathbf{q}^{(i)}(T)=0, \text { in } \Omega,\left.\quad \mathbf{q}^{(i)}\right|_{\Sigma}=0
\end{array}\right.
$$

Multiplying (3.13) by $\mathbf{z}^{(i)}$, performing an integration by parts, and using (3.12), we obtain

$$
\alpha_{i}\left(\mathbf{u}-\mathbf{u}_{i, d}, \mathbf{z}^{(i)}\right)_{\omega_{i, d} \times(0, T)}=\left(\mathbf{q}^{(i)}, \partial_{t} \mathbf{z}^{(i)}-\nu \Delta \mathbf{z}^{(i)}\right)_{Q}=\left(\mathbf{q}^{(i)}, \mathbf{v}^{(i)}\right)_{\omega_{i} \times(0, T)} .
$$


Setting the above identity in (3.11), we have

$$
\left(\mathbf{q}^{(i)}, \mathbf{v}^{(i)}\right)_{\omega_{i} \times(0, T)}+\mu_{i}\left(\overline{\mathbf{v}}^{(i)}, \mathbf{v}^{(i)}\right)_{\omega_{i} \times(0, T)}=0, \quad \forall \mathbf{v}^{(i)} \in \mathbf{L}^{2}\left(\omega_{i} \times(0, T)\right) ;
$$

hence we arrive at

$$
\overline{\mathbf{v}}^{(i)}=-\frac{1}{\mu_{i}} \mathbf{q}^{(i)} \chi_{\omega_{i}}, \quad i=1,2,
$$

which jointly with (3.13) and the state system generate the optimality system (3.10).

\section{ON THE APPROXIMATE CONTROLLABILITY PROBLEM}

In this section, we give a result of the approximate controllability type when a Stackelberg-Nash strategy is used. Such a result will imply, in particular, that Problem 2 is well defined, because the corresponding admissible set is nonempty.

First of all, we will define a generic Nash problem:

Problem $(P)_{\text {aux }}$ :

Given $\mathbf{h} \in \mathbf{L}^{2}(\Omega)$, we consider the following auxiliary multi-objective problem: To optimize the two functionals $(i=1,2)$

$$
I_{i}\left(\mathbf{w}^{(1)}, \mathbf{w}^{(2)}\right)=\frac{\alpha_{i}}{2} \int_{0}^{T} \int_{\omega_{i}}|\mathbf{y}|^{2} d x d t+\frac{\mu_{i}}{2} \int_{0}^{T} \int_{\omega_{i, d}}\left|\mathbf{w}^{(i)}\right|^{2} d x d t,
$$

with respect to $\left(\mathbf{w}^{(1)}, \mathbf{w}^{(2)}\right)$ in the Nash sense, with the state $\mathbf{y}$ solution of the problem:

$$
\left\{\begin{array}{l}
-\partial_{t} \mathbf{y}-\nu \Delta \mathbf{y}+\nabla s=\mathbf{w}^{(1)} \chi_{\omega_{1, d}}+\mathbf{w}^{(2)} \chi_{\omega_{2, d}}, \text { in } Q \\
\operatorname{div} \mathbf{y}=0 \text { in } Q ; \\
\mathbf{y}(T)=\mathbf{h}, \text { in } \Omega ;\left.\quad \mathbf{y}\right|_{\Sigma}=0 .
\end{array}\right.
$$

Lemma 4.1 (Existence, uniqueness and characterization of Nash problem $\left.(P)_{\text {aux }}\right)$.

(i) Assume that

$$
\alpha_{1}\left\|M_{2}\right\|^{2}<4 \mu_{2} \quad \text { and } \quad \alpha_{2}\left\|M_{1}\right\|^{2}<4 \mu_{1},
$$

where the operators $M_{i}$ are defined in (4.5) below. Then, for any $\mathbf{h} \in \boldsymbol{L}^{2}(\Omega)$, one has existence and uniqueness of the Nash equilibrium problem $(P)_{\text {aux }}$.

(ii) Given $\mathbf{h} \in \boldsymbol{L}^{2}(\Omega)$, the Nash equilibrium $\left(\overline{\mathbf{w}}^{(1)}, \overline{\mathbf{w}}^{(2)}\right)$ for $(P)_{\text {aux }}$ is characterized by $\overline{\mathbf{w}}^{(i)}=\alpha_{i} \mathbf{p}^{(i)} \chi_{\omega_{i, d}}, i=1,2$, where $\left(\mathbf{y}, \mathbf{p}^{(1)}, \mathbf{p}^{(2)}\right)$ satisfy the coupled system of Stokes type:

$$
\left\{\begin{array}{l}
-\partial_{t} \mathbf{y}-\nu \Delta \mathbf{y}+\nabla s=\alpha_{1} \mathbf{p}^{(1)} \chi_{\omega_{1, d}}+\alpha_{2} \mathbf{p}^{(2)} \chi_{\omega_{2, d}}, \text { in } Q \\
\partial_{t} \mathbf{p}^{(i)}-\nu \Delta \mathbf{p}^{(i)}+\nabla \delta^{(i)}=-\frac{1}{\mu_{i}} \mathbf{y} \chi_{\omega_{i}}, \text { in } Q \\
\operatorname{div} \mathbf{y}=\operatorname{div} \mathbf{p}^{(i)}=0 \text { in } Q ; \\
\mathbf{y}(T)=\mathbf{h}, \mathbf{p}^{(i)}(0)=0, \text { in } \Omega ;\left.\quad \mathbf{y}\right|_{\Sigma}=\left.\mathbf{p}^{(i)}\right|_{\Sigma}=0
\end{array}\right.
$$

Proof. First of all, we show $(i)$; i.e., there exists a unique Nash equilibrium for the problem $(P)_{\text {aux }}$. Recall that $\mathcal{H}_{i}=L^{2}\left(\omega_{i, d} \times(0, T)\right), i=1,2, \mathcal{H}=\mathcal{H}_{1} \times \mathcal{H}_{2}$ and we consider the operators $M_{i} \in \mathcal{L}\left(\mathcal{H}_{i}, \mathbf{L}^{2}(Q)\right)$ defined as

$$
M_{i} \mathbf{w}^{(i)}=\mathbf{z}^{(i)},
$$


where $\left(\mathbf{z}^{(i)}, r_{i}, \mathbf{w}^{(i)}\right)$ satisfy the problem

$$
\left\{\begin{array}{l}
-\partial_{t} \mathbf{z}^{(i)}-\nu \Delta \mathbf{z}^{(i)}+\nabla r^{(i)}=\mathbf{w}^{(i)} \chi_{\omega_{i, d}}, \text { in } Q ; \\
\operatorname{div} \mathbf{z}^{(i)}=0 \text { in } Q ; \\
\mathbf{z}^{(i)}(T)=0, \text { in } \Omega ;\left.\quad \mathbf{z}^{(i)}\right|_{\Sigma}=0 .
\end{array}\right.
$$

Notice that $\mathbf{z}^{(i)}$ is the derivative of the state $\mathbf{y}$ with respect to $\mathbf{w}^{(i)}$.

Thus, we can write the solution of the state equation (4.2) as

$$
\mathbf{y}=M_{1} \mathbf{w}^{(1)}+M_{2} \mathbf{w}^{(2)}-G,
$$

where $G$ is the solution of the problem

$$
\left\{\begin{array}{l}
-\partial_{t} G-\nu \Delta G+\nabla P=0, \text { in } Q ; \\
\operatorname{div} G=0 \text { in } Q ; \\
G(T)=-\mathbf{h}, \text { in } \Omega ;\left.\quad G\right|_{\Sigma}=0 .
\end{array}\right.
$$

With this notation, we can rewrite the functionals defined in (4.1) as follows:

$$
\begin{aligned}
I_{i}\left(\mathbf{w}^{(1)}, \mathbf{w}^{(2)}\right) & =\frac{\alpha_{i}}{2} \int_{0}^{T} \int_{\omega_{i}}\left|M_{1} \mathbf{w}^{(1)}+M_{2} \mathbf{w}^{(2)}-G\right|_{2}^{2} d x d t \\
& +\frac{\mu_{i}}{2} \int_{0}^{T} \int_{\omega_{i, d}}\left|\mathbf{w}^{(i)}\right|_{2}^{2} d x d t .
\end{aligned}
$$

Consequently, $\left(\overline{\mathbf{w}}^{(1)}, \overline{\mathbf{w}}^{(2)}\right)$ is a Nash equilibrium for $(P)_{a u x}$ if and only if

$$
\left\langle\frac{\partial I_{i}}{\partial \mathbf{w}^{(i)}}\left(\overline{\mathbf{w}}^{(1)}, \overline{\mathbf{w}}^{(2)}\right), \mathbf{w}^{(i)}\right\rangle=0, \quad i=1,2, \quad \forall\left(\mathbf{w}^{(1)}, \mathbf{w}^{(2)}\right),
$$

which yields

$$
\alpha_{i}\left(M_{1} \overline{\mathbf{w}}^{(1)}+M_{2} \overline{\mathbf{w}}^{(2)}-G, M_{i} \mathbf{w}^{(i)}\right)_{\omega_{i} \times(0, T)}+\mu_{i}\left(\overline{\mathbf{w}}^{(i)}, \mathbf{w}^{(i)}\right)_{\omega_{i, d} \times(0, T)}=0,
$$

for all $\left(\mathbf{w}^{(1)}, \mathbf{w}^{(2)}\right)$ and $i=1,2$; hence

$$
\alpha_{i} M_{i}^{*}\left[\left(M_{1} \overline{\mathbf{w}}^{(1)}+M_{2} \overline{\mathbf{w}}^{(2)}\right) \chi_{\omega_{i}}\right]+\mu_{i} \overline{\mathbf{w}}^{(i)} \chi_{\omega_{i, d}}=M_{i}^{*}\left(G \chi_{\omega_{i}}\right), i=1,2,
$$

where $M_{i}^{*} \in \mathcal{L}\left(\mathbf{L}^{2}(Q), \mathcal{H}_{i}\right)$ is the adjoint operator of $M_{i}$. The rest of the proof follows an analogous form of the proof of Proposition 3.1

Now, we prove the characterization $(i i)$. We know that $\left(\overline{\mathbf{w}}^{(1)}, \overline{\mathbf{w}}^{(2)}\right)$ is a Nash equilibrium for $\left(P_{\text {aux }}\right)$ if and only if (4.10) holds, that is,

$$
\alpha_{i}\left(\mathbf{y}, \mathbf{z}^{(i)}\right)_{\omega_{i} \times(0, T)}+\mu_{i}\left(\overline{\mathbf{w}}^{(i)}, \mathbf{w}^{(i)}\right)_{\omega_{i, d} \times(0, T)}=0 \quad(i=1,2),
$$

where $\left(\mathbf{z}^{(i)}, r_{i}\right)$ is the solution of the system (4.6). In order to write (4.12) only as a function of $\mathbf{w}^{(i)}$, we consider the adjoint system

$$
\left\{\begin{array}{l}
\partial_{t} \mathbf{p}^{(i)}-\nu \Delta \mathbf{p}^{(i)}+\nabla \delta^{(i)}=-\frac{1}{\mu_{i}} \mathbf{y} \chi_{\omega_{i}}, \text { in } Q ; \\
\operatorname{div} \mathbf{p}^{(i)}=0 \text { in } Q ; \\
\mathbf{p}^{(i)}(0)=0, \text { in } \Omega ; \text { and }\left.\mathbf{p}^{(i)}\right|_{\Sigma}=0 .
\end{array}\right.
$$

Multiplying (4.13) by $\mathbf{z}^{(i)}$, integrating by parts and using (4.6), we can arrive at

$$
-\frac{1}{\mu_{i}}\left(\mathbf{y}, \mathbf{z}^{(i)}\right)_{\omega_{i} \times(0, T)}=\left(\mathbf{p}^{(i)}, \mathbf{w}^{(i)}\right)_{\omega_{i, d} \times(0, T)} .
$$

From the identities (4.12) and (4.14), we obtain

$$
-\alpha_{i} \mu_{i}\left(\mathbf{p}^{(i)}, \mathbf{w}^{(i)}\right)_{\omega_{i, d} \times(0, T)}+\mu_{i}\left(\overline{\mathbf{w}}^{(i)}, \mathbf{w}^{(i)}\right)_{\omega_{i, d} \times(0, T)}=0, i=1,2
$$


for each $\mathbf{w}^{(i)}$; hence (since $\left.\mu_{i}>0\right)$,

$$
\overline{\mathbf{w}}^{(i)}=\alpha_{i} \mathbf{p}^{(i)} \chi_{\omega_{i, d}}, i=1,2,
$$

and part $(i i)$ of the proof is completed.

In order to prove the approximate controllability of problem 1 we will need the following unique continuation result:

Lemma 4.2. Assume $\omega_{1} \cup \omega_{2} \subset \omega$. If $\left(\mathbf{y}, \mathbf{p}^{(1)}, \mathbf{p}^{(2)}\right)$ is a solution of problem (4.4) such that $\mathbf{y} \equiv 0$ in $\omega \times(0, T)$. Then $\left(\mathbf{y}, \mathbf{p}^{(1)}, \mathbf{p}^{(2)}\right) \equiv 0$ in $Q$.

Proof. From the hypothesis $\omega_{i} \subset \omega(i=1,2)$, we have in particular that $\mathbf{y} \equiv 0$ in $\left(\omega_{1} \cup \omega_{2}\right) \times(0, T)$; hence the right-hand side of the system for $\mathbf{p}^{(i)}$ in (4.4) vanishes. Therefore, $\mathbf{p}^{(i)} \equiv 0$ in $Q$. Substituting this result in the first equation of system (4.4), we obtain that $(\mathbf{y}, s)$ is a solution of the Stokes problem

$$
\left\{\begin{array}{l}
-\partial_{t} \mathbf{y}-\nu \Delta \mathbf{y}+\nabla s=0, \text { in } Q \\
\operatorname{div} \mathbf{y}=0 \text { in } Q ; \\
\mathbf{y}(T)=\mathbf{h}, \text { in } \Omega ;\left.\quad \mathbf{y}\right|_{\Sigma}=0
\end{array}\right.
$$

Therefore, the Unique Continuation Theorem for the Stokes problem [6] implies that

$$
\mathbf{y} \equiv 0 \quad \text { in } Q
$$

Now, we are in a position to prove the approximate controllability result.

Theorem 4.3. Suppose hypotheses (3.5) and (4.3) hold (in particular, for each $\mathbf{f} \in L^{2}(\omega \times(0, T))$, and Problem 1 has a unique solution $\left.\left(\overline{\mathbf{v}}^{(1)}(\mathbf{f}), \overline{\mathbf{v}}^{(2)}(\mathbf{f})\right)\right)$. Then, the set

$$
\left\{\mathbf{u}(T)=\mathbf{u}\left(\cdot, T ; \mathbf{f}, \overline{\mathbf{v}}^{(1)}, \overline{\mathbf{v}}^{(2)}\right) \in \boldsymbol{H} ; \mathbf{f} \in L^{2}(\omega \times(0, T))\right\}
$$

is a dense subset of $\boldsymbol{H}$.

Remark 4.4. For instance, both hypotheses (3.5) and (4.3) are verified if we change $\left\|L_{i}\right\|^{2}$ and $\left\|M_{i}\right\|^{2}$ in (3.5) and (4.3) respectively by $\widetilde{C}>0$, a constant such that $\|S(\mathbf{e})\|_{L^{2}(Q)} \leq \widetilde{C}\|\mathbf{e}\|_{L^{2}(Q)}$, where $S(\mathbf{e})$ is the velocity solution of the Stokes problem with right-hand side equal to $\mathbf{e} \in \mathbf{L}^{2}(Q)$.

Proof. Firstly, we decompose the Nash equilibrium as follows:

$$
\left(\mathbf{u}, \mathbf{q}^{(1)}, \mathbf{q}^{(2)}\right)=\left(\widetilde{\mathbf{u}}, \widetilde{\mathbf{q}}^{(1)}, \widetilde{\mathbf{q}}^{(2)}\right)+\left(\widehat{\mathbf{u}}, \widehat{\mathbf{q}}^{(1)}, \widehat{\mathbf{q}}^{(2)}\right),
$$

where $\left(\widetilde{\mathbf{u}}, \widetilde{\mathbf{q}}^{(1)}, \widetilde{\mathbf{q}}^{(2)}\right)$ depends on $\left(\mathbf{g}, \mathbf{u}_{1, d}, \mathbf{u}_{2, d}, \mathbf{u}_{0}\right)$ via the problem

$$
\left\{\begin{array}{l}
\partial_{t} \widetilde{\mathbf{u}}-\nu \Delta \widetilde{\mathbf{u}}+\nabla \widetilde{p}=\mathbf{g}-\frac{1}{\mu_{1}} \widetilde{\mathbf{q}}^{(1)} \chi_{\omega_{1}}-\frac{1}{\mu_{2}} \widetilde{\mathbf{q}}^{(2)} \chi_{\omega_{2}}, \text { in } Q ; \\
-\partial_{t} \widetilde{\mathbf{q}}^{(i)}-\nu \Delta \widetilde{\mathbf{q}}^{(i)}+\nabla \widetilde{r}_{i}=\alpha_{i}\left(\mathbf{u}_{0}-\mathbf{u}_{i, d}\right) \chi_{\omega_{i, d}} \text { in } Q \quad(i=1,2) ; \\
\operatorname{div} \widetilde{\mathbf{u}}=0, \text { in } Q \operatorname{div} \widetilde{\mathbf{q}}^{(i)}=0, \text { in } Q \quad(i=1,2) ; \\
\widetilde{\mathbf{u}}(0)=\mathbf{u}_{0}, \text { in } \Omega,\left.\widetilde{\mathbf{u}}\right|_{\Sigma}=0, \\
\widetilde{\mathbf{q}}^{(i)}(T)=0, \text { in } \Omega,\left.\widetilde{\mathbf{q}}^{(i)}\right|_{\Sigma}=0 \quad(i=1,2),
\end{array}\right.
$$


and $\left(\widehat{\mathbf{u}}, \widehat{\mathbf{q}}^{(1)}, \widehat{\mathbf{q}}^{(2)}\right)$ depends on $\mathbf{f}$ via the problem

$$
\left\{\begin{array}{l}
\partial_{t} \widehat{\mathbf{u}}-\nu \Delta \widehat{\mathbf{u}}+\nabla \widehat{p}=\mathbf{f} \chi_{\omega}-\frac{1}{\mu_{1}} \widehat{\mathbf{q}}^{(1)} \chi_{\omega_{1}}-\frac{1}{\mu_{2}} \widehat{\mathbf{q}}^{(2)} \chi_{\omega_{2}}, \text { in } Q ; \\
-\partial_{t} \widehat{\mathbf{q}}^{(i)}-\nu \Delta \widehat{\mathbf{q}}^{(i)}+\nabla \widehat{r}^{(i)}=\alpha_{i} \widehat{\mathbf{u}} \chi_{\omega_{i, d}} \text { in } Q \quad(i=1,2) ; \\
\operatorname{div} \widehat{\mathbf{u}}=0, \text { in } Q ; \operatorname{div} \widehat{\mathbf{q}}^{(i)}=0, \text { in } Q \quad(i=1,2) ; \\
\widehat{\mathbf{u}}(0)=0, \text { in } \Omega,\left.\widehat{\mathbf{u}}\right|_{\Sigma}=0, \\
\widehat{\mathbf{q}}^{(i)}(T)=0, \text { in } \Omega,\left.\widehat{\mathbf{q}}^{(i)}\right|_{\Sigma}=0 \quad(i=1,2) .
\end{array}\right.
$$

Notice that the two previous problems have a unique solution, because they are particular cases of problem (3.10).

Secondly, since $\left(\widetilde{\mathbf{u}}, \widetilde{\mathbf{q}}^{(1)}, \widetilde{\mathbf{q}}^{(2)}\right)$ is fixed (independently of $\mathbf{f}$ ), it suffices to prove the density of the set furnished by $\widehat{\mathbf{u}}(T)$ in $\mathbf{H}$. For this, let $\mathbf{h}$ be a given function in $\mathbf{H}$ and suppose

$$
(\widehat{\mathbf{u}}(T), \mathbf{h})_{\Omega}=0, \quad \forall \mathbf{f} \in \mathbf{L}^{2}(\omega \times(0, T)) .
$$

Then, it suffices to show that $\mathbf{h} \equiv 0$.

Let $\left\{\mathbf{y}, s, \mathbf{p}^{(i)}, \delta^{(i)}\right\}$ be the unique solution of system (4.4) associated to $\mathbf{h}$, which exists thanks to Lemma 4 and hypothesis (4.3). Taking as test functions $\widehat{\mathbf{u}}, \widehat{\mathbf{q}}^{(1)}$ and $\widehat{\mathbf{q}}^{(2)}$ in the respective equations of the system (4.4) and performing integrations by parts, taking into account (4.19), we get

$$
\begin{aligned}
& \left(\alpha_{1} \mathbf{p}^{(1)} \chi_{\omega_{1, d}}+\alpha_{2} \mathbf{p}^{(2)} \chi_{\omega_{2, d}}, \widehat{\mathbf{u}}\right)_{Q}=-(\mathbf{h}, \widehat{\mathbf{u}}(T))_{\Omega} \\
& +\left(\mathbf{y}, \mathbf{f} \chi_{\omega}-\frac{1}{\mu_{1}} \widehat{\mathbf{q}}^{(1)} \chi \omega_{1}-\frac{1}{\mu_{2}} \widehat{\mathbf{q}}^{(2)} \chi \omega_{2}\right)_{Q}
\end{aligned}
$$

and

$$
\left(-\frac{1}{\mu_{i}} \mathbf{y} \chi_{\omega_{i}}, \widehat{\mathbf{q}}^{(i)}\right)_{Q}=\alpha_{i}\left(\mathbf{p}^{(i)}, \widehat{\mathbf{u}}\right)_{\omega_{i, d} \times(0, T)} .
$$

Adding the identity (4.22) for $i=1,2$,

$$
\left(\mathbf{y},-\frac{1}{\mu_{1}} \widehat{\mathbf{q}}^{(1)} \chi_{\omega_{1}}-\frac{1}{\mu_{2}} \widehat{\mathbf{q}}^{(2)} \chi_{\omega_{2}}\right)_{Q}=\left(\alpha_{1} \mathbf{p}^{(1)} \chi_{\omega_{1, d}}+\alpha_{2} \mathbf{p}^{(2)} \chi_{\omega_{2, d}}, \widehat{\mathbf{u}}\right)_{Q}
$$

Comparing identities (4.21) and (4.23), we obtain

$$
(\mathbf{h}, \widehat{\mathbf{u}}(T))_{\Omega}=\left(\mathbf{y}, \mathbf{f} \chi_{\omega}\right)_{Q}, \quad \forall \mathbf{f} \in \mathbf{L}^{2}(\omega \times(0, T)) .
$$

From the above identity and the condition (4.20), we have

$$
\mathbf{y} \equiv 0 \quad \text { in } \omega \times(0, T) \text {. }
$$

Then, the unique continuation result given in Lemma 4.2 implies that

$$
\mathbf{y} \equiv 0 \quad \text { in } Q .
$$

In particular, $\mathbf{h} \equiv 0$. This completes the proof.

Remark 4.5. Let us observe that the result of approximate controllability given in Theorem 4.3 is related to the unique continuation property of the adjoint problem (4.4) given in Lemma 4.2. One possible question arises: is it possible to obtain the null controllability (which is stronger than approximate controllability) of the Stackelberg-Nash strategies for Stokes equations? More precisely, is it possible to obtain $\overline{\mathbf{f}}$ such that $\mathbf{u}(T):=\mathbf{u}\left(\cdot, T ; \overline{\mathbf{f}}, \overline{\mathbf{v}}^{(1)}, \overline{\mathbf{v}}^{(2)}\right)=0$ ? The answer is not obvious, because this null controllability must be related to an observability inequality of 
the adjoint problem (4.4); i.e. given $\mathbf{y}(T)=\mathbf{h}$, one should bound in appropriate weighted spaces a norm of $\left(\mathbf{y}, \mathbf{p}^{(1)}, \mathbf{p}^{(2)}\right)$ only by a norm of $\mathbf{y} \chi_{\omega_{1} \cup \omega_{2}}$.

\section{THE ACTION OF THE OPTIMAL LEADER}

For each $\mathbf{f}$, let $\left(\overline{\mathbf{v}}^{(1)}(\mathbf{f}), \overline{\mathbf{v}}^{(2)}(\mathbf{f})\right)$ be the Nash equilibrium solution of Problem 1 and $\alpha>0$ be a given number. We will show that there exists an optimal leader control $\overline{\mathbf{f}}$ solution of the following problem:

$$
\inf _{\mathbf{f} \in L^{2}(\omega \times(0, T))}\left\{\frac{1}{2} \int_{0}^{T} \int_{\omega}|\mathbf{f}|^{2} d x d t ; \mathbf{u}\left(\cdot, T ; \mathbf{f}, \overline{\mathbf{v}}^{(1)}, \overline{\mathbf{v}}^{(2)}\right) \in B_{L^{2}}\left(\mathbf{u}_{T}, \alpha\right)\right\} .
$$

Note that this minimization problem is well-posed because the corresponding feasible set is not empty, owing to Theorem 4.3 .

\section{Theorem 5.1.}

(i) The dual minimization problem

$$
\inf _{\mathbf{h} \in L^{2}(\Omega)}\left\{\frac{1}{2} \int_{0}^{T} \int_{\omega}|\mathbf{y}|^{2} d x d t+\alpha\|\mathbf{h}\|_{L^{2}(\Omega)}-\int_{\Omega} \mathbf{h} \cdot \mathbf{u}_{T} d x\right\}
$$

has a unique solution.

(ii) The minimum $\overline{\mathbf{f}}$ of problem (5.1) is characterized by $\overline{\mathbf{f}}=\mathbf{y} \chi_{\omega}$, where $\left(\mathbf{y}, \mathbf{p}^{(1)}, \mathbf{p}^{(2)}\right)$ is the solution of (4.4) associated to $\mathbf{h}$ given as the solution of (5.2) $)$

Notice that the problem (5.1) has a unique solution as a consequence of (i) and (ii).

Proof. To prove (ii), we consider the following maps $F: \mathbf{f} \in L^{2}((0, T) \times \omega) \rightarrow \mathbb{R}$ and $G: \mathbf{h} \in L^{2}(\Omega) \rightarrow \overline{\mathbb{R}}$ defined as

$$
\begin{gathered}
F(\mathbf{f})=\frac{1}{2} \int_{0}^{T} \int_{\omega}|\mathbf{f}|^{2} d x d t, \\
G(\mathbf{h})= \begin{cases}0 & \text { if } \mathbf{h}+\widetilde{\mathbf{u}}(T) \in B_{L^{2}}\left(\mathbf{u}_{T}, \alpha\right) ; \\
+\infty & \text { if } \mathbf{h}+\widetilde{\mathbf{u}}(T) \in L^{2}(\Omega) \backslash B_{L^{2}}\left(\mathbf{u}_{T}, \alpha\right),\end{cases}
\end{gathered}
$$

where $\widetilde{\mathbf{u}}(t)$ is the solution of (4.18). Then problem (5.1) is equivalent to the problem

$$
\inf _{\mathbf{f} \in L^{2}(\omega \times(0, T))}\{F(\mathbf{f})+G(L \mathbf{f})\},
$$

where $L: \mathbf{f} \in L^{2}((0, T) \times \omega) \rightarrow L^{2}(\Omega)$ is the linear functional defined as $L \mathbf{f}=$ $\widehat{\mathbf{u}}\left(\cdot, T ; \mathbf{f}, \overline{\mathbf{v}}^{(1)}, \overline{\mathbf{v}}^{(2)}\right)$ and where $\widehat{\mathbf{u}}$ is the solution of (4.19). By the duality theory of Fenchel and Rockafellar (see [15]), we have

$$
\inf _{\mathbf{f} \in L^{2}(\omega \times(0, T))}\{F(\mathbf{f})+G(L \mathbf{f})\}=\sup _{\mathbf{h} \in L^{2}(\Omega)}\left\{F^{*}\left(L^{*} \mathbf{h}\right)+G^{*}(-\mathbf{h})\right\},
$$

where $L^{*}$ is the adjoint operator of $L$ and $F^{*}$ is the polar function of $F$, that is,

$$
F^{*}(\mathbf{y})=\sup _{\mathbf{y}^{*} \in L^{2}(\omega \times(0, T))}\left\{\int_{0}^{T} \int_{\omega} \mathbf{y} \cdot \mathbf{y}^{*} d x d t-F\left(\mathbf{y}^{*}\right)\right\} .
$$

From (4.4) we obtain the identity (4.24), i.e. $(\mathbf{h}, \widehat{\mathbf{u}}(T))_{\Omega}=\left(\mathbf{y}, \mathbf{f} \chi_{\omega}\right)_{Q}$ for any $\mathbf{f}$. Then

$$
\left(L^{*} \mathbf{h}, \mathbf{f}\right)_{\Omega}=(\mathbf{h}, L \mathbf{f})_{\Omega}=(\mathbf{h}, \widehat{\mathbf{u}}(T))_{\Omega}=\left(\mathbf{y}, \mathbf{f} \chi_{\omega}\right)_{Q}=\left(\mathbf{y} \chi_{\omega}, \mathbf{f}\right)_{Q}, \quad \forall \mathbf{f}
$$


Therefore, we deduce

$$
L^{*} \mathbf{h}=\mathbf{y} \chi_{\omega}
$$

It is well known ([5]) that

$$
F^{*}(\mathbf{y})=\frac{1}{2} \int_{0}^{T} \int_{\omega}|\mathbf{y}|^{2} d x d t
$$

and

$$
G^{*}(\mathbf{h})=\alpha\|\mathbf{h}\|_{L^{2}(\Omega)}+\int_{\Omega} \mathbf{h} \cdot \mathbf{u}_{T} d x
$$

hence we can deduce $(i i)$.

To prove $(i)$, we use the analogous arguments of [3], 4. We observe that given $\mathbf{h} \in L^{2}(\Omega)$, Lemma 4 implies that there exists a unique solution to the system (4.4). Obviously, the functional

$$
R(\mathbf{h})=\frac{1}{2} \int_{0}^{T} \int_{\omega}|\mathbf{y}|^{2} d x d t+\alpha\|\mathbf{h}\|_{L^{2}(\Omega)}-\int_{\Omega} \mathbf{h} \cdot \mathbf{u}_{T} d x
$$

is convex and continuous. Now, we are going to prove that the functional $R$ is coercive, in fact that $R$ satisfies

$$
\liminf _{\|\mathbf{h}\|_{L^{2}(\Omega)} \rightarrow \infty} \frac{R(\mathbf{h})}{\|\mathbf{h}\|_{L^{2}(\Omega)}} \geq \alpha .
$$

To prove (5.4), let us consider a sequence $\left(\mathbf{h}_{j}\right)$ in $L^{2}(\Omega)$ with $\left\|\mathbf{h}_{j}\right\|_{L^{2}(\Omega)} \rightarrow \infty$ and define the normalized sequence

$$
\widehat{\mathbf{h}}_{j}=\frac{\mathbf{h}_{j}}{\left\|\mathbf{h}_{j}\right\|_{L^{2}(\Omega)}} .
$$

By using Lemma 4, we obtain by linearity that $\mathbf{y}_{j}=\left\|\mathbf{h}_{j}\right\|_{L^{2}(\Omega)} \widehat{\mathbf{y}}_{j}$, where $\mathbf{y}_{j}=$ $\mathbf{y}\left(\cdot, \cdot ; \mathbf{h}_{j}\right)$ and $\widehat{\mathbf{y}}_{j}=\mathbf{y}\left(\cdot, \cdot ; \widehat{\mathbf{h}}_{j}\right)$. Consequently, we get

$$
\begin{aligned}
\frac{R\left(\mathbf{h}_{j}\right)}{\left\|\mathbf{h}_{j}\right\|_{L^{2}(\Omega)}} & =\frac{\left\|\mathbf{h}_{j}\right\|_{L^{2}(\Omega)}}{2} \int_{0}^{T} \int_{\omega}\left|\widehat{\mathbf{y}}_{j}\right|^{2} d x d t+\alpha\left\|\widehat{\mathbf{h}}_{j}\right\|_{L^{2}(\Omega)}-\int_{\Omega} \widehat{\mathbf{h}}_{j} \cdot \mathbf{u}_{T} d x \\
& \geq \frac{\left\|\mathbf{h}_{j}\right\|_{L^{2}(\Omega)}}{2} \int_{0}^{T} \int_{\omega}\left|\widehat{\mathbf{y}}_{j}\right|^{2} d x d t+\alpha-\left\|\mathbf{u}_{T}\right\|_{L^{2}(\Omega)} .
\end{aligned}
$$

Now, we consider two cases:

- Case 1: Assume

$$
\liminf _{j \rightarrow \infty} \int_{0}^{T} \int_{\omega}\left|\widehat{\mathbf{y}}_{j}\right|^{2} d x d t>0 .
$$

From (5.5), we have

$$
\liminf _{j \rightarrow \infty} \frac{R\left(\mathbf{h}_{j}\right)}{\left\|\mathbf{h}_{j}\right\|_{L^{2}(\Omega)}}=+\infty .
$$

- Case 2: Assume

$$
\liminf _{j \rightarrow \infty} \int_{0}^{T} \int_{\omega}\left|\widehat{\mathbf{y}}_{j}\right|^{2} d x d t=0 .
$$


In this case, we consider a convergent subsequence, which we denote the same way as the sequence, such that

$$
\int_{0}^{T} \int_{\omega}\left|\widehat{\mathbf{y}}_{j}\right|^{2} d x d t \rightarrow 0 \quad \text { as } j \rightarrow \infty
$$

and

$$
\widehat{\mathbf{h}}_{j} \rightarrow \mathbf{h} \text { weakly in } L^{2}(\Omega), \quad \text { as } j \rightarrow \infty .
$$

Using (5.7), the solution of system (4.4), $\widehat{\mathbf{y}}_{j}=\mathbf{y}\left(\cdot, \cdot ; \widehat{\mathbf{h}}_{j}\right)$ converges to $\mathbf{y}(\cdot, \cdot ; \mathbf{h})$ for any given $\mathbf{h}$. Therefore, owing to (5.6),

$$
\mathbf{y}=0 \text { in } \omega \times(0, T) \text {. }
$$

Then, Lemma 4.2 implies that

$$
\mathbf{y} \equiv 0 \quad \text { in } Q .
$$

In particular, $\mathbf{h} \equiv 0$ in $\Omega$ and, consequently, from (5.7),

$$
\widehat{\mathbf{h}}_{j} \rightarrow 0 \text { weakly in } L^{2}(\Omega), \quad \text { as } j \rightarrow \infty .
$$

Taking the limit in (5.5), we infer that (5.4) is verified.

Finally, since $R$ is strictly convex, $R$ has a unique critical point that is the minimum of $R$.

\section{ACKNOWLEDGMENT}

We thank the referee for a careful reading of the manuscript and for valuable comments.

\section{REFERENCES}

[1] Dautray, R., Díaz, J. I., Agir Pour Conserver L'environnement?: Réflexions Générales et Analyse Mathématique de Deux Problèmes Concrets, Notes from the XIth Jacques-Louis Lions Hispano-French School on Numerical Simulation in Physics and Engineering (Spanish), 77-118, Grupo Anal. Teor. Numer. Modelos Cienc. Exp. Univ. Cádiz, Cádiz, 2004.

[2] Díaz, J. I., Lions, J. L., On the Approximate Controllability of Stackelberg-Nash Strategies, in Ocean Circulation and Pollution Control. A Mathematical and Numerical Inquiry (J.I. Díaz, ed.). Lecture Notes, Proceedings of the Diderot video-conference, Amsterdam-Madrid-Venice, Lecture Notes, Springer Verlag, pp. 17-28, 2004. MR2026005

[3] Díaz, J. I., On the von Neumann problem and the approximate controllability of StackelbergNash strategies for some environmental problems, Rev. Real Acad. Cienc. Serie A Mat., V. 96 (3), 343-356, 2002. MR1985740 (2004e:93011)

[4] Díaz, J. I., Ramos, A. M., Positive and negative approximate controllability results for semilinear parabolic equations, Rev. Real Acad. Cienc. Exact. Fis. y Nat., LXXXIX, 11-30, 1995. MR $1454346(98 \mathrm{e}: 93007)$

[5] Ekeland, I., Temam, R., Analyse convexe et problèmes variationnels. (French) Collection Études Mathématiques. Dunod; Gauthier-Villars, 1974. MR0463993 (57:3931a)

[6] Fernández-Cara, E., Guerrero, S., Imanuvilov, O. Yu., Puel, J.-P., Local exact controllability of the Navier-Stokes system, J. Math. Pures Appl. 12 (9), Vol. 83 (2004), 1501-1542. MR2103189 (2005g:93013)

[7] Gayte, I., Guillén-González, F., Marques-Lopes, F. P., Rojas-Medar, M. A., Optimal Control and PDE, Workshop sobre "Avances recientes en el análisis y control de ecuaciones diferenciales no lineales". Anal. Control Ec. Dif. No Lin., 1 (2004), 125-141.

[8] Glowinski, R., Ramos, A. M., Periaux, J., Nash equilibria for the multiobjective control of linear partial differential equations, J. Optim. Theory Appl. 112 (2002), no. 3, 457-498. MR.1892232 (2003e:49041) 
[9] Ramos, A. M., Glowinski, R., Periaux, J., Pointwise Control of the Burgers Equation and Related Nash Equilibrium Problems: Computational Approach, Journal of Optimization Theory and Applications, Vol. 112, pp. 499-516, 2002. MR1892233 (2003e:93043)

[10] Lions, J. L., Contrôle de Pareto de Systèmes Distribués. Le cas stationnaire. C.R. Acad. Sc. Paris, t. 302, Série I, n ${ }^{0}$ 6, 1986, 223-227. MR832049 (87d:93043)

[11] Lions, J. L., Contrôle de Pareto de Systèmes Distribués. Le cas d'évolution. C.R. Acad. Sc. Paris, t. 302, Série I, ${ }^{0}$ 11, 1986, 413-417. MR838591 (87e:49011)

[12] Lions, J. L., Some Remarks on Stackelberg's Optimization, Mathematical Models and Methods in Applied Sciences, Vol. 4, pp. 477-487, 1994. MR1291134 (96c:93133)

[13] Nash, J. F., Non-cooperative games, Annals of Mathematics (2), 54, pp. 286-295, 1951. MR0043432 (13:261g)

[14] Pareto, V., Cours d'économie politique, Rouge, Laussane, Switzerland, 1896.

[15] Rockafellar, R. T., Duality and stability in extremum problems involving convex functions, Pacific. J. of Math., 21, 167-187, 1967. MR0211759(35:2636)

[16] Von Stackelberg, H., Marktform und Gleichgewicht, Springer, Berlin, Germany, 1934.

[17] Temam, R., Navier-Stokes equations. Theory and Numerical Analysis. North-Holland, 1984. MR769654 (86m:76003)

Departamento de Ecuaciones Diferenciales y Análisis Numérico, Universidad de Sevilla, Aptdo. 1160, 41080 Sevilla, Spain

E-mail address: guillen@us.es

Departamento de Matemática, UFPA, CP 479, 66075-110, Belém-PA, Brazil

E-mail address: fpmlopes@ufpa.br

GMa-Departamento de Ciencias Básicas, Universidad del Bío-Bío, Facultad de CienCias, Campus Fernando May, Casilla 447, Chillán, Chile

E-mail address: marko@ueubiobio.cl 